\title{
Estimates on the non-compact expanding gradient Ricci solitons
}

\author{
Xiang Gao, Qiaofang Xing and Rongrong Cao
}

\begin{abstract}
In this paper, we deal with the complete non-compact expanding gradient Ricci soliton $\left(M^{n}, g\right)$ with positive Ricci curvature. On the condition that the Ricci curvature is positive and the scalar curvature approaches 0 towards infinity, we derive a useful estimate on the growth of potential functions. Based on this and under the same assumptions, we prove that $\int_{0}^{t} R c\left(\gamma^{\prime}(s), \gamma^{\prime}(s)\right) d s$ and $\int_{0}^{t} R c\left(\gamma^{\prime}(s), \nu\right) d s$ at least have linear growth, where $\gamma(s)$ is a minimal normal geodesic emanating from the point where $R$ obtains its maximum. Furthermore, some other results on the Ricci curvature are also obtained.
\end{abstract}

\section{Introduction and Main Results}

Ricci solitons are fixed points of the Ricci flow as a dynamical system on the space of Riemannian metrics modulo diffeomorphisms and scalings. From the equation point of view, they are natural generalizations of the Einstein metrics. In this paper, in particular we study the expanding gradient Ricci solitons and the definition is as follows:

Definition 1.1. A complete Riemannian manifold $\left(M^{n}, g\right)$ is called an expanding gradient Ricci soliton if there is a smooth function $f: M^{n} \rightarrow \mathbb{R}$, such that

$$
R c+\nabla \nabla f=\lambda g,
$$

Key Words: Ricci flow, expanding gradient Ricci soliton, Ricci curvature.

2010 Mathematics Subject Classification: Primary 53C25; Secondary 53C44.

Received: January, 2013.

Revised: April, 2013.

Accepted: April, 2013.

This work is supported by the National Natural Science Foundation of China (NSFC) 11301493, 11101267 and Fundamental Research Funds for the Central Universities. 
where $R c$ is the Ricci curvature tensor and $\lambda$ is a negative real number.

Recall that gradient Ricci solitons are the most widely studied Ricci solitons, and quite a few results on the classification of gradient Ricci solitons have appeared. In particular, if $\left(M^{n}, g\right)$ is compact, then by the maximum principle, it is elementary to check that $f$ in (1) has to be a constant so that the expanding gradient Ricci soliton $M^{n}$ is actually an Einstein manifold with negative Ricci curvature. Hence in this paper, we are only interested in the non-compact case, on the condition that the Ricci curvature is positive and the scalar curvature approaches 0 towards infinity, we derive a useful estimate on the growth of the potential function $f$ as follows:

Theorem 1.2. If $\left(M^{n}, g\right)$ is a complete expanding gradient Ricci soliton with positive Ricci curvature and the scalar curvature approaches 0 towards infinity, then for any sufficient small $\varepsilon>0$, there exists $r_{0}$ such that when $r(x)>r_{0}$, we have

$$
\begin{aligned}
& -\frac{1}{2 \lambda}\left(\frac{\lambda}{1+\varepsilon}\left(r(x)-r_{0}\right)-\sqrt{R_{0}+2 \lambda f\left(x_{0}\right)}\right)^{2}+\frac{1}{2 \lambda} R_{0} \\
\leq & -f(x) \\
\leq & -\frac{1}{2 \lambda}\left(\left(\lambda r(x)-\sqrt{R_{0}}\right)^{2}-R_{0}\right),
\end{aligned}
$$

where $r_{0}=\operatorname{dist}\left(O, x_{0}\right)$.

By using Theorem 1.2, we can also derive an estimate on the integral $\int_{0}^{t} R c\left(\gamma^{\prime}(s), \gamma^{\prime}(s)\right) d s$ as follows:

Theorem 1.3. If $\left(M^{n}, g\right)$ is a complete expanding gradient Ricci soliton with positive Ricci curvature and the scalar curvature approaches 0 towards infinity, then for any sufficient small $\varepsilon>0$, there is $r_{0}$ such that when $r(x)>r_{0}$, we have

$$
\begin{aligned}
& \int_{0}^{t} R c\left(\gamma^{\prime}(s), \gamma^{\prime}(s)\right) d s \\
\geq & -\frac{1}{2 \lambda r(x)}\left(\frac{\lambda}{1+\varepsilon}\left(r(x)-r_{0}\right)-\sqrt{R_{0}+2 \lambda f\left(x_{0}\right)}\right)^{2}+\frac{1}{2 \lambda r(x)} R_{0} \\
& +\lambda r(x),
\end{aligned}
$$

where $\gamma(s)$ is a minimal normal geodesic emanating from $O, x=\gamma(t)$ and $r_{0}=\operatorname{dist}\left(O, x_{0}\right)$. 
Remark 1. Theorem 1.3 states that the integral $\int_{0}^{t} R c\left(\gamma^{\prime}(s), \gamma^{\prime}(s)\right) d s$ as long as the geodesic emanates from the origin is independent on the choice of a particular geodesic and only dependent on the end point $x$. Moreover we also have another similar result as follows:

Theorem 1.4. If $\left(M^{n}, g\right)$ is a complete expanding gradient Ricci soliton with positive Ricci curvature and the scalar curvature approaches 0 towards infinity, then any sufficient small $\varepsilon>0$, there is $r_{0}$ such that when $r(x)>r_{0}$, we have

$$
\begin{aligned}
& \int_{0}^{t} R c\left(\gamma^{\prime}(s), \nu(s)\right) d s \\
\geq & \sqrt{\left(\frac{\lambda}{1+\varepsilon}\left(r(x)-r_{0}\right)-\sqrt{R_{0}+2 \lambda f\left(x_{0}\right)}\right)^{2}-\left(\lambda r(x)-\sqrt{R_{0}}\right)^{2}} \\
& +\lambda r(x),
\end{aligned}
$$

where $\gamma(s)$ is a minimal normal geodesic emanating from $O, \nu=-\frac{\nabla f}{|\nabla f|}$ and $r_{0}=\operatorname{dist}\left(O, x_{0}\right)$.

The paper is organized as follows: In section 2, we derive a technical lemma and then prove Theorem 1.2. In section 3, we derive another useful lemma and present the proof of Theorem 1.3 and 1.4.

\section{Lemmas and Proof of Theorem 1.2}

In this section, we firstly show that if there is any maximum point of scalar curvature $R$, then it is unique.

Lemma 2.1. If $\left(M^{n}, g\right)$ is a complete expanding gradient Ricci soliton with positive Ricci curvature, then there is at most one maximum point of the scalar curvature $R$.

Proof. On the expanding gradient Ricci soliton we have (see [4])

$$
\nabla R=2 R c(\nabla f, \cdot),
$$

in particular at a critical point $p$, where $\nabla R(p)=0$ we have

$$
0=\langle\nabla R(p), \nabla f(p)\rangle=2 R c(\nabla f(p), \nabla f(p)) .
$$

Then by strict positivity of the Ricci curvature we have $\nabla f(p)=0$. Furthermore, since

$$
\nabla \nabla f=\lambda g-R c<0
$$

for the expanding gradient Ricci soliton, we conclude that $p$ is the unique maximum point of the potential function $f$. 
By Morse theory, Lemma 2.1 shows that $M^{n}$ is diffeomorphic to the Euclidean space $\mathbb{R}^{n}$. Moreover if we assume that the Ricci curvature is positive and the scalar curvature $R$ approaches 0 towards spatial infinity, then there must be at least one point where $R$ obtains its maximum. By Lemma 2.1 we see that the point of maximum is unique. We denote $O$ as the point of maximum of $R$, called the origin, and assume $f(O)=0$ by adding a constant and $R(O)>1$ by multiplying a constant. On the expanding gradient Ricci soliton, we also have (see [4]):

$$
R+|\nabla f|^{2}-2 \lambda f=C
$$

thus

$$
R+|\nabla f|^{2}-2 \lambda f=R(O)=R_{0} .
$$

For any $x \in M^{n}$, let $r(x)=\operatorname{dist}(O, x)$ and $\gamma(s)$ denote the shortest geodesic from $O$ to $x$, where $s$ is the arclength. Now we give the proof of Theorem 1.2.

Proof of Theorem 1.2. By (4) and positivity of $R$ we have

$$
|\nabla f|^{2}=R_{0}-R+2 \lambda f<R_{0}+2 \lambda f,
$$

thus

$$
\left|\nabla \sqrt{R_{0}+2 \lambda f}\right|=\frac{\left|\nabla\left(R_{0}+2 \lambda f\right)\right|}{2 \sqrt{R_{0}+2 \lambda f}}<-\lambda .
$$

Along any geodesic $\gamma(s)$ emanating from $O$ we have

$$
\begin{aligned}
\left|\frac{d}{d s} \sqrt{R_{0}+2 \lambda f}(\gamma(s))\right| & =\left|\left\langle\nabla \sqrt{R_{0}+2 \lambda f}, \gamma^{\prime}(s)\right\rangle\right| \\
& \leq\left|\nabla \sqrt{R_{0}+2 \lambda f}\right| \\
& <-\lambda .
\end{aligned}
$$

Notice that the maximum of $f$ is 0 so that $f<0$, we deduce that

$$
\left|\sqrt{R_{0}+2 \lambda f(x)}-\sqrt{R_{0}}\right| \leq-\lambda r(x)
$$

By using $\lambda<0$, we have

$$
-f(x) \leq-\frac{1}{2 \lambda}\left(\left(\lambda r(x)-\sqrt{R_{0}}\right)^{2}-R_{0}\right)
$$

For the lower bound of $f(x)$, we work on the integral curve of $-\frac{\nabla f}{|\nabla f|^{2}}$, which is denoted by $\beta(\sigma)$. Since 


$$
\frac{d f(\beta(\sigma))}{d \sigma}=\left\langle\nabla f, \beta^{\prime}(\sigma)\right\rangle=-\left\langle\nabla f, \frac{\nabla f}{|\nabla f|^{2}}\right\rangle=-1,
$$

together with $f(O)=0$ we have $f(\beta(\sigma))=-\sigma$.

Since the scalar curvature $R$ approaches 0 towards spatial infinity, we have

$$
|\nabla f| \rightarrow \sqrt{R_{0}+2 \lambda f}
$$

as $x \rightarrow \infty$. Then given any sufficient small $\varepsilon$ such that $0<\varepsilon<\sqrt{R_{0}}-1$, there is $\sigma_{0}$ such that when $\sigma \geq \sigma_{0}$, we have

$$
|\nabla f(\beta(\sigma))|>\sqrt{R_{0}-2 \lambda \sigma}-\varepsilon
$$

along the integral curve $\beta(\sigma)$.

Let $x_{0}=\beta\left(\sigma_{0}\right)$, then the length of $\beta$ from $x_{0}$ to $x$ can be estimated as

$$
\begin{aligned}
\int_{\sigma_{0}}^{\sigma}\left|\beta^{\prime}(\sigma)\right| d \sigma & =\int_{\sigma_{0}}^{\sigma} \frac{1}{|\nabla f|} d \sigma \\
& <\int_{\sigma_{0}}^{\sigma} \frac{1}{\sqrt{R_{0}-2 \lambda \sigma}-\varepsilon} d \sigma \\
& <\int_{\sigma_{0}}^{\sigma} \frac{1+\varepsilon}{\sqrt{R_{0}-2 \lambda \sigma}} d \sigma
\end{aligned}
$$

where we use $0<\varepsilon<\sqrt{R_{0}}-1$. Thus

$$
\begin{aligned}
\int_{\sigma_{0}}^{\sigma}\left|\beta^{\prime}(\sigma)\right| d \sigma & <-\frac{1+\varepsilon}{\lambda}\left(\sqrt{R_{0}-2 \lambda \sigma}-\sqrt{R_{0}-2 \lambda \sigma_{0}}\right) \\
& =-\frac{1+\varepsilon}{\lambda}\left(\sqrt{R_{0}+2 \lambda f(x)}-\sqrt{R_{0}+2 \lambda f\left(x_{0}\right)}\right) .
\end{aligned}
$$

On the other hand,

$$
\int_{\sigma_{0}}^{\sigma}\left|\beta^{\prime}(\sigma)\right| d \sigma \geq \operatorname{dist}\left(x, x_{0}\right)=r(x)-r_{0},
$$

where $r_{0}=\operatorname{dist}\left(O, x_{0}\right)$, so we have

$$
-\frac{1+\varepsilon}{\lambda}\left(\sqrt{R_{0}+2 \lambda f(x)}-\sqrt{R_{0}+2 \lambda f\left(x_{0}\right)}\right) \geq r(x)-r_{0} .
$$

Hence

$$
-f(x) \geq-\frac{1}{2 \lambda}\left(\sqrt{R_{0}+2 \lambda f\left(x_{0}\right)}-\frac{\lambda}{1+\varepsilon}\left(r(x)-r_{0}\right)\right)^{2}+\frac{1}{2 \lambda} R_{0}
$$


Remark 2. Substituting (5) into (4), we can also get that:

Corollary 2.2. Under the same assumptions of Theorem 1.2, we have

$$
R(x) \leq R_{0}+2 \lambda f(x) \leq\left(\lambda r(x)-\sqrt{R_{0}}\right)^{2}
$$

\section{Proof of Theorem 1.3 and 1.4}

In this section, we present the proof of Theorem 1.3 and 1.4. Let $\nu=-\frac{\nabla f}{|\nabla f|}$ and $\theta(x)$ denote the angle between $\gamma^{\prime}(x)$ and $\nu$, using the notations of last section we can prove the following result:

Lemma 3.1. $\theta(x) \leq \frac{\pi}{3}$ as $x \rightarrow \infty$.

Proof. Since

$$
\nabla \nabla f=\lambda g-R c<0,
$$

we see that $-f$ is geodesically convex and furthermore

$$
\frac{d}{d s}(-f(\gamma(s))) \geq \frac{-f(x)+f(O)}{r(x)}=\frac{-f(x)}{r(x)}
$$

On the other hand

$$
\frac{d}{d s}(-f(\gamma(s)))=\left\langle-\nabla f, \gamma^{\prime}\right\rangle=|\nabla f|\left\langle\nu, \gamma^{\prime}\right\rangle=|\nabla f| \cos \theta(x)
$$

and using (5) it follows that

$$
|\nabla f| \leq \sqrt{R_{0}+2 \lambda f}<\sqrt{R_{0}}-\lambda r(x)
$$

Hence we have

$$
\begin{aligned}
\cos \theta(x) \geq & \frac{1}{|\nabla f|} \cdot \frac{-f(x)}{r(x)} \\
\geq & -\frac{1}{2 \lambda r(x)\left(\sqrt{R_{0}}-\lambda r(x)\right)}\left(\sqrt{R_{0}+2 \lambda f\left(x_{0}\right)}-\frac{\lambda}{1+\varepsilon}\left(r(x)-r_{0}\right)\right)^{2} \\
& +\frac{R_{0}}{2 \lambda r(x)\left(\sqrt{R_{0}}-\lambda r(x)\right)} .
\end{aligned}
$$

Let $x \rightarrow \infty$ we get $\cos \theta(x) \geq \frac{1}{2}$, and the result follows.

Then by using Theorem 1.2 and Lemma 3.1, we can now prove Theorem 1.3 . 
Proof of Theorem 1.3. Along a minimal geodesic $\gamma(s)$ we have

$$
\frac{d}{d s}(|\nabla f| \cos \theta)=\gamma^{\prime}\left\langle-\nabla f, \gamma^{\prime}\right\rangle=\left\langle-\nabla_{\gamma^{\prime}} \nabla f, \gamma^{\prime}\right\rangle=R c\left(\gamma^{\prime}, \gamma^{\prime}\right)-\lambda
$$

As the proof of Lemma 3.1 we have

$$
|\nabla f| \cos \theta(x) \geq \frac{-f(x)}{r(x)}
$$

taking integral from 0 to $x$ along $\gamma(s)$ and using (6) we get

$$
\begin{aligned}
\int_{0}^{t} R c\left(\gamma^{\prime}(s), \gamma^{\prime}(s)\right) d s & =\int_{0}^{t}\left(\frac{d}{d s}(|\nabla f| \cos \theta)+\lambda\right) d s \\
& =|\nabla f| \cos \theta(x)-|\nabla f| \cos \theta(0)+\lambda r(x) \\
& \geq \frac{-f(x)}{r(x)}+\lambda r(x) \\
\geq & -\frac{1}{2 \lambda r(x)}\left(\frac{\lambda}{1+\varepsilon}\left(r(x)-r_{0}\right)-\sqrt{R_{0}+2 \lambda f\left(x_{0}\right)}\right)^{2} \\
& +\frac{1}{2 \lambda r(x)} R_{0}+\lambda r(x)
\end{aligned}
$$

where we use $x=\gamma(t)$.

Now we turn to prove Theorem 1.4.

Proof of Theorem 1.4. As the proof of Theorem 1.3, instead of $|\nabla f| \cos \theta$ we take derivative of $|\nabla f|$ along the geodesic $\gamma(s)$, and get

$$
\frac{d}{d s}|\nabla f|=\frac{1}{2|\nabla f|} \gamma^{\prime}\langle\nabla f, \nabla f\rangle=\left\langle-\nabla_{\gamma^{\prime}} \nabla f, \nu\right\rangle=R c\left(\gamma^{\prime}, \nu\right)-\lambda \cos \theta
$$

Taking integral from 0 to $x$ along $\gamma(s)$ and using (6) and (7) we get

$$
\begin{aligned}
& \int_{0}^{t} R c\left(\gamma^{\prime}(s), \nu(s)\right) d s=\int_{0}^{t}\left(\frac{d}{d s}|\nabla f|+\lambda \cos \theta\right) d s \\
& \geq|\nabla f|(x)-|\nabla f|(0)+\lambda \int_{0}^{t} d s \\
& =\sqrt{R_{0}-R+2 \lambda f}+\lambda r(x) \\
& \geq \sqrt{\left(\frac{\lambda}{1+\varepsilon}\left(r(x)-r_{0}\right)-\sqrt{R_{0}+2 \lambda f\left(x_{0}\right)}\right)^{2}} \\
& \overline{-\left(\lambda r(x)-\sqrt{R_{0}}\right)^{2}}+\lambda r(x) \text {. }
\end{aligned}
$$




\section{References}

[1] S. Brendle, R. Schoen, Manifolds with 1/4-pinched curvature are space forms, J. Amer. Math. Soc. 22 (2009), 287-307.

[2] H. W. Chen, Pointwise quarter-pinched 4-manifolds, Ann. Global Anal. Geom. 9 (1991), 161-176.

[3] B. Chow, S. C. Chu, D. Glickenstein, C. Guentheretc, J. Isenberg, T. Ivey, D. Knopf, P. Lu, F. Luo and L. Ni, The Ricci flow: techniques and applications. Part II: analytic aspects. Mathematical Surveys and Monographs, 144, AMS, Providence, RI, (2008).

[4] Bennett Chow, Peng Lu, Lei Ni, Hamilton's Ricci Flow. Lectures in Contemporary Mathematics, 3, Science Press and Graduate Studies in Mathematics, 77, American Mathematical Society (co-publication), 2006.

[5] R. S. Hamilton, Four manifolds with positive curvature operator, J. Diff. Geom., 24, (1986), 153-179.

[6] Grisha Perelman, The entropy formula for the Ricci flow and its geometric applications, arXiv:math.DG/0211159.

Xiang Gao,

School of Mathematical Sciences,

Ocean University of China,

Lane 238, SongLing Road, Laoshan District, Qingdao City,

Shandong Province, 266100, People's Republic of China.

Email: gaoxiangshuli@126.com

Qiaofang Xing,

Institute of Science,

Information Engineering University,

Zhengzhou City, Henan Province, 450001, People's Republic of China.

Rongrong Cao,

School of Mathematical Sciences,

Qingdao University,

Lane 308, Ningxia Road, Shinan District, Qingdao City,

Shandong Province, 266071, People's Republic of China. 